The cognitive and behavioral effects of chronic epilepsy have been emphasized in several publications by Deonna T (cited in Deonna T. Childhood epilepsy: secondary prevention is crucial. Dev Med Child Neurol Aug 2003;45 (suppl 95):38-41). Some focal epilepsies or purely EEG paroxysmal discharges without clinical seizures can have a chronic adverse effect on cognition and behavior. Treatment should sometimes be directed not only to the control of clinical seizures but also the prevention of cognitive impairments related to focal epileptiform discharges, as for example, epilepsy with so-called benign rolandic spikes. The risks of cognitive decline related to chronic AED treatment are increasingly apparent, especially with temporal lobe epilepsies, emphasizing the importance of early referral of selected patients to our surgical colleagues.

\title{
PROGNOSTIC VALUE OF EEG IN FOCAL SEIZURE CONTROL
}

Patients aged 2 to 85 (mean 32) years whose focal seizures were uncontrolled (U) (more than 2 seizures per month) and a group with controlled seizures (C) (fewer than 2 seizures per year), 150 in each group, were randomly selected from files at the University of Illinois Medical Center, Chicago, and EEGs in the two groups (804 in U group and 674 in $\mathrm{C}$ group) were examined for their predictive value of seizure control. Patients with generalized epilepsy and benign epilepsies of childhood were excluded. Younger patients (5-18 yrs) were generally better controlled than those 21-48 years of age, and seizure intractability became more prevalent in the 20 to 40 year age group. Focal spikes and focal slow waves were more common in $\mathrm{U}$ patients than $\mathrm{C}$ patients. Many spikes or slow waves occurred in EEGs of $\mathrm{U}$ patients and no or rare spikes or slow waves in C patients' records. Predictors of $\mathrm{C}$ focal seizures were no or rare spikes, especially on the first or second EEG. $\mathrm{U}$ patients were correctly identified by many spikes (at least one/10 sec) at any location or frontal spikes in $84 \%$ cases ( $61 \%$ with 1 st or 2 nd EEG), but $29 \%\left(21 \%\right.$ in $1^{\text {st }}$ or $\left.2^{\text {nd }} E E G s\right)$ were incorrectly identified as $U$ and seizures were controlled. The EEG is a useful predictor of prognosis of the uncontrolled focal seizure patient, and should be a guide to early more effective therapy. (Hughes JR, Fino JJ. Focal seizures and EEG: prognostic considerations. Clin Electroencephalogr October 2003;34:174-181). (Reprints: John R Hughes MD, PhD, University of Illinois Medical Center M/C 796, $912 \mathrm{~S}$ Wood St, Chicago, IL 60612).

COMMENT. Based on frequent spike occurrence in first or second EEG recordings in patients with focal seizures, a positive prediction of uncontrolled seizures and poor prognosis is likely in $61 \%$ but a false positive may occur in $21 \%$. In any EEG recording, the true positives are $84 \%$ and false positives $29 \%$. Seizure intractability is especially frequent in the 20 to 40 year age group. Patients with rare spikes have less frequent less severe focal seizures and a lower incidence of secondarily generalized tonicclonic seizures (Rosati A et al. Neurology 2003;60:1290-1295; cited by Hughes). 